\title{
Subtypes of Developmental Coordination Disorder: Research on Their Nature and Etiology
}

\author{
Laurence Vaivre-Douret \\ University of Paris Descartes and Inserm Unit UMR-SO 669 University of Paris-Sud and \\ Paris Descartes; AP-HP Necker-Enfants Malades, Child Psychiatry Department and Port \\ Royal-Cochin, Paris, France \\ Christophe Lalanne \\ Inserm Unit UMR-SO 669 University of Paris-Sud and Paris Descartes, Paris, France \\ Isabelle Ingster-Moati
}

University of Paris 7 Denis Diderot and Inserm Unit UMR-SO 669 University of Paris-Sud and Paris Descartes; AP-HP Necker-Enfants Malades, Opthalmology Department, Paris, France

Nathalie Boddaert

University of Paris Descartes and Inserm Unit 797 Orsay; AP-HP Necker-Enfants Malades, Child Neuro-radiology Department, Paris, France

Dominique Cabrol

AP-HP Port Royal-Cochin Hospital, Department of Obstetrics and Gynecology, Paris, France

Jean-Louis Dufier

University of Paris Descartes; AP-HP Necker-Enfants Malades, Opthalmology Department, Paris, France

Bernard Golse

University of Paris Descartes and Inserm Unit UMR-SO 669 University of Paris-Sud and Paris Descartes; AP-HP Necker-Enfants Malades, Child Psychiatry Department and Port Royal-Cochin, Department of Obstetrics and Gynecology, Paris, France

\section{Bruno Falissard}

Inserm Unit UMR-SO 669 University of Paris-Sud and Paris Descartes; University of Paris-Sud, Department of Biostatistics, AP-HP Villejuif, France

Correspondence should be addressed to Professor Laurence Vaivre-Douret, AP-HP Necker-Enfants Malades, Unité 669 INSERM, 149 rue de Sèvres, 75743 Paris Cedex 15, France. E-mail: laurence.vaivre-douret@inserm.fr 
Children with Developmental Coordination Disorder (DCD) are a group embracing clumsiness and developmental dyspraxia. Our study provides a better understanding of the nature of DCD and its etiology, and identifies subtypes of dyspraxia. Forty-three children with DCD (5-15 years) were enrolled on the Diagnostic and Statistical Manual of Mental Disorders (4th ed. [DSM-IV-TR]; American Psychiatric Association, 2000) criteria. Extensive standardized evaluations were conducted. We distinguished from two patterns of "pure" developmental dyspraxia: ideomotor and visual-spatial/visual-constructional, and mix dyspraxia with more co-morbidities. Our study provides a better understanding of the nature of DCD, and sheds light on its etiology and brain dysfunction, so as to identify subtypes of developmental DCD/dyspraxia with specific clinical criteria.

Descriptions of children with mild motor coordination deficits and clumsy movements have been discussed in the literature since the early 1900s, for instance by Collier who referred to "congenital clumsiness" (Ford, 1966; for a review, Vaivre-Douret, 2007). In 1969, a French Child psychiatrist and psychologist (Ajuriaguerra (de) \& Stambak, 1969) described "child dyspraxia," by reference to "constructional apraxia" of adults, and defined it as a disorder of body integration interfering with spatial organization. Ayres (1972) and Gubbay (1979) who at first used the term "developmental apraxia" later preferred to use the term "dyspraxia." The term "developmental dyspraxia" was applied to children falling into the category described as "clumsy" (e.g. Lesny, 1980). "Developmental dyspraxia" also appeared in the literature with the work of Denckla (1984), or Cermak (1985). Dyspraxia is also presented as a constitutional developmental disorder involving an impairment in learning or in performing non-habitual motor tasks typically identified in children. This is defined as a failure to have ever acquired the ability to perform given age-appropriate complex motor actions or voluntary motor activities activity, in absence of physical and/or neurological disorder, or general intellectual retardation. In contrast, "apraxia" is an acquired disorder that leads to the loss in the ability to accomplish previously learned skills.

Thus, dyspraxia is not a simple version of adult apraxia, which most commonly consists of difficulties in planning and execution of complex movement sequences in adults with brain damage, where the cortical lesion is often located in the left parietal lobe, in the pre-motor cortex, in the supplementary motor area (e.g., De Lange, Hagoort, \& Toni, 2005) or in the frontal cortex in association with language impairments such as aphasia. However, there is a difficulty in medical and scientific communities in defining dyspraxia because varying terminologies are used in the literature to describe children with coordination difficulties (for a review, see Missiuna \& Polatajko, 1995).

It thus appears that dyspraxia and DCD are often regarded as synonymous. The term DCD was the first to be introduced by the American Psychiatric Association in the Diagnosis and Statistical Manual, Revised third edition in 1987 and recently in the DSM-IV and DSM-IV-TR revised in 2000.

Children with DCD represent a significant proportion of school-age children. DCD is a common disorder with an estimated prevalence of $6 \%$ between 5 and 11 years of age, with higher incidence among boys than girls (American Psychiatric Association, 2000).

Children with DCD are a heterogeneous group, embracing clumsiness and developmental dyspraxia. DCD appears as a collection of conditions without any clearly defined clinical signs and symptoms or any specific disorder as found in the definitions of other developmental 
disorders like Autism or attention deficit disorder. This lack of diagnostic specificity does not assist understanding of the interplay between the different mechanisms and processes in DCD.

\section{ETIOLOGY}

The etiology of developmental dyspraxia and DCD is unknown but seems to be connected with maturational processes in the central nervous system. However, no consensus has been established, while heterogeneous causes are suggested: prematurity, impairment of dominant cerebral hemisphere, sensory integration disorder, perinatal factors consecutive to anoxia or to hypoxia. Other proposed causes have involved inter or intra hemispheric connection disorders (Geschwind, 1975), cortex, cerebellar or basal ganglia dysfunction (Lundy-Ekman, Ivry, Keele, \& Woollacott, 1991), parietal dysfunction (Lesny, 1980). A high incidence has been noted of non-specific cerebral abnormalities in positron emission tomography (PET) or in magnetic resonance imagery (MRI), with cortical atrophy or demyelinization (e.g., Knuckey, Apsimon, \& Gubbay, 1983), thinning of the corpus callosum and moderate ventricular dilatation (Vaivre-Douret, 2002). Oculo-motor or gaze disorders have also been observed (Mazeau, 1995 ) and neuro-visual abnormalities have been shown in some studies on coordination disorders (Sigmundsson, Hansen, \& Talcott, 2003; Ingster-Moati et al., 2005). It is also well known (Grönqvist, Gredeback, \& Hofsten, 2006) that the posterior parietal and superior temporal lobe regions are involved in smooth pursuit movement (SP) and the networks for vertical SP mature later than those for horizontal SP (Ingster-Moati et al., 2009). The study by Mon-Williams, Mackie, McCulloch, and Pascal (1996) on the visually evoked potentials (VEP) in 14 children with DCD, allowing for inattention and movement artefacts, showed no significant differences between the control group and the DCD group times, but significantly smaller VEP amplitude in children with DCD.

\section{ATTEMPTS AT CLASSIFICATION}

In studies on series of analytical factors, Ayres (1972) defined the nature of the disorder in developmental dyspraxia as a sensory integration problem characterized by difficulties in planning movement sequences and not just in executing them. This author identified deficits in processing somato-sensory information with respect to tactile and kinaesthetic perception and she suggested that the dorsal column medial lemniscal system seems to be involved in fine motor tasks, whereas the vestibular system is more involved in gross motor performance.

According to Cermark (1985), Roy's classification (1978) for apraxia in adults seems to describe the typology of dyspraxic children. For Cermark (1985), there are two types of dyspraxic disorder: motor planning disorder and executive disorder. The results of Ayres's work (1972) pointed to an integration disorder that seems to correspond to a deficit in motor planning, such as secondary planning dyspraxia disorder. Dewey (1991) has shown in a study that children with sensory-motor dysfunction demonstrated deficits in the performance of symbolic representational limb gestures (more for transitive than intransitive gestures) and action sequences, both on verbal command and by imitation. These results show an ideomotor and ideational disorder similar to the category of apraxic adults corresponding to planning dyspraxia (ideo-motor dyspraxia). 
An attempt at classification (Albaret, Carayre, Soppelsa, \& Michelon, 1995) involved four groups of French dyspraxic children in a multiple correspondence factorial analysis. The first group obtained is characterized by lack of fine manual coordination and dysgraphia, the second is associated with a lack of manual coordination, constructive and ideo-motor praxic disorders, slowness and synkinesis disorders. The third group contains the most acute disturbances with a general impairment in all study variables. The fourth group was associated with a lack of manual coordination, synkinesis disorders, balance disorders and slowness. Thus the reporting on particular types of impairment may be influenced by the characteristics chosen by researchers to measure them (Missiuna, \& Polatajko, 1995).

\section{CLUSTER ANALYSIS}

Some recent studies have used cluster analysis as a method to identify distinct subtypes of children with DCD. In this approach a set of measurements is acquired and subjects are grouped together on the basis of the profiles of their scores. However, the subtypes reported by these studies differed with respect to the variables or measures included, the numbers and characteristics of the subjects and the statistical methods. These methodological choices can be important for the interpretation of the cluster structures obtained (for a review, see Macnab, Miller, \& Polatajko, 2001). For example, Lyytinen and Ahonen (1988) tested motor control, global motor coordination (balance and jump), fine motor coordination, synkinesis (co-movement and mirror movements), kinaesthetic perception, and visual-spatial perception (VMI, Beery, 1982), and also they identified six clusters. Miyahara (1994) used only the gross motor subtests from the Bruininks Oseretsky Test of Motor Profiency (BOTMP, Bruininks, 1978) and Wright and Sugden (1996) from the M-ABC Test (Henderson \& Sugden, 1992), and found four subtypes. Despite differences in the variables used in the cluster analysis, studies on subtypes of DCD mainly used measures of performance on gross motor skills (Visser, 2003), except for Lyytinen and Ahonen (1988), Dewey and Kaplan (1994), Hoare (1994) and Macnab and colleagues (2001) who also used perceptual motor measures, and Dewey and Kaplan (1994) who are the only researchers to have used transitive gestures with motor sequencing.

There emerges from this research a set of standardized motor assessments typically used by researchers and clinicians: M-ABC (Henderson, \& Sugden, 1992), BOTMP (Polatajko, \& Cantin, 2005), and TOMI (Stott, Moyes, \& Henderson, 1984) (the M-ABC being particularly widely used). They have become de facto standards because of the frequency of their use in research and practice. Although these measures are used to identify children suspected of DCD, results of a comparative study have shown that the M-ABC identifies more children as having DCD than the BOTMP. However, the M-ABC test does not enable appreciation of specific neurodevelopmental markers of brain system development because sensory-motor function, and motor milestones are regarded as screening indices of general neural integrity and not as indices of neuro-developmental processes. Such test batteries are useful, but it is very difficult in studies on DCD subtypes to interpret the results solely on motor-based performances. The type and cause of impairment needs to be analysed by means of neuromuscular examination. In addition, visual-motor perceptual performance is not widely investigated.

It is regrettable that co-morbidities and soft neurological signs have not been considered in most of the studies on DCD so as to enable a better understanding of the semiology and etiology 
of DCD. Indeed, according Rasmussen and Gillbert (2000) and to Kaplan, Wilson, Dewey, and Crawford (1998) in the DCD population, co-morbidity (attention deficity hyperactivity disorder [ADHD], specific language impairment) tends to be the rule rather than the exception. As underlined by Macnab and colleagues (2001) in the literature there are many inconsistencies in the descriptions of DCD and it is not known whether they are due to sample variation or to the existence of discrete subtypes (Rourke, 1985).

Studies on subtypes of DCD do not sufficiently take account of soft neurological signs pointing to the areas of cerebral involvement. There is indeed evidence of cerebellar involvement (for a review, see Visser, 2003). An integrative model proposed was by Vaivre-Douret (2007) in a first clinical approach, in order to understand the various stages in cerebral processes and their structural involvement in developmental dyspraxia.

In summary, a few studies have investigated different functions together in DCD children, such as neuromuscular tone and specific difficulties-academic, language, gnosic, visualmotor/visual-perceptual, and executive-in children exhibiting different types of sensory-motor deficit. Such investigations are useful if they differentiate between normal and deviant children, but the type and cause of deviation needs to be analysed by means of neuromuscular tone examination. A complete investigation using different measures of function could provide a better understanding of the nature of DCD, and could cast light on mechanisms and etiology, and on associated brain dysfunction, so as to identify subtypes of developmental DCD/dyspraxia with specific clinical criteria.

\section{METHOD}

\section{Participants}

This study was conducted by Inserm Unit 669 in the out-patient consultation of the Child Psychiatry Department, Necker Hospital, Paris. Informed consent (parents and children) to participate was mandatory. Institutional review board approval was obtained for the investigations. A sample of 43 children with dyspraxia or DCD aged between 5 and 15 years $(M=8.31$; $S D=2.39)$ comprising 8 girls $(19 \%)$ and 35 boys $(81 \%)$ were enrolled between September 2005 and January 2007. They were initially selected on DSM-IV-R criteria from a clinical population of children from the Paediatric Department of Port Royal-Cochin Hospital and from the Child Psychiatry Department of Necker Hospital, France. They were screened during a first outpatient clinic consultation for mild to moderate motor-coordination difficulties interfering with the performance of daily activities (Criterion A, DSM-IV-R, American Psychiatric Association, 2000) and with academic achievement (Criterion B, DSM-IV-R), but without psychopathology trouble. Children were included in the study if they had not been previously assessed, or managed by an occupational therapist, and were not taking medication. Ineligibility criteria were as follows: visual or auditory deficit, neurological impairment (including traumatic brain injury), ADHD, or any other psychiatric abnormalities (even minor ones), preterm birth $(<37$ weeks). Overall, 43 children were identified as having DCD following DSM-IV-R criteria. Participants, whose parents' socioeconomic status (SES) range from middle to high level, were all Caucasians. However, SES and ethnicity were not considered as exclusion criteria. 


\section{Procedure and Testing Materials}

At first, based on our previous clinical trial (Vaivre-Douret, 2007), questions were asked of parents on the following: pregnancy and delivery (birth term, abnormalities); age of the first motor acquisitions (sitting alone, crawling, walking alone, first sentences). Visual refraction disorders presented by the child (myopia, astygmatism, hypermetropia) and disorders such as repetitive otitis, were noted. Difficulties with block-building or constructive manipulatory play, such as Lego blocks, following a model and completion of puzzles, as reported by parents, were also noted. Learning disabilities based on school failure in certain academic performances (such as arithmetic, handwriting) were noted from school reports. In the second phase, extensive neuro-psychological and neuro-psychomotor evaluations with neuro-visual examination were administered and high-resolution cerebral anatomical MRI was performed. Various cerebral functions were assessed using published tests administered in accordance with standardized instructions. All test scores were standardized following authors' scoring guidelines. They all are expressed as either deviation from population mean in standard deviation units (failure if $<1 S D$ ) or as a score distribution (failure if $<20$ th or 30 th percentile age-adjusted, depending on the test under consideration). It should be noted that the percentile-based cutoffs used throughout this study are rather large, which might lead to a higher prevalence for some of the reported impairments in this particular sample. Although the use of such cutoffs only apply to three tests, this might limit the generalizability of our findings on a wider population where other cutoff may be applied. For most of the tests, cutoff scores varied with age.

Neuro-psychological assessment. In the neuro-psychological assessment, all children completed all subtests of a standard Wechsler measure of intelligence. Other specific standardized tests were administered: visual-perceptual-motor tests such as visual constructional skills (reproduction of a block design, Khos, 1972), visual-spatial structuring (manual copy followed by visual-spatial memory of a complex geometric figure, Rey (1959) and the Visual-Motor Integration test (VMI, Beery, 1982)), which requires the child to make a manual copy of a series of 24 geometric drawings of progressively increasing difficulty. The visual-spatial attention was assessed with a bell-crossing test (Odédys, 2005) similar to that proposed by Gauthier, Dehaut, and Joanette (1989). Mental executive functions (mental planning) were assessed with the Porteus Labyrinth test (1952), and the Tower of London test by Korkman, Kirk, and Kemp (2003). A handwriting scale was used to detect dysgraphy by assessing the quality of gesture and the regularity and form of letters (Ajuriaguerra, Auzias, \& Denne, 1989). A score of 19 to 25 points indicates a significant abnormality. We also assessed visual perception (visual gnosis) tasks (recognizing forms, Frostig, 1973; tangled lines and the naming of animals seen in outline from the rear) (Rey, 1941), language screening battery (Odédys, 2005; N-EEL, ChevrieMuller, \& Plaza, 2001), which included tasks of reading, repetition of words and logatoms, picture-naming speed, meta-phonological tests, auditory memory and working memory tasks (digit span). We added a "status test" to examine kinaesthetic perception (memory), positioning the child's arm and finger and asking him with eyes closed to remember and repeat. Both had to be successful to score positive. In addition, during all tests we noted whether or not the child was restless, suggesting hyperkinesia.

Neuro-psychomotor assessment. The neuro-psychomotor evaluation consists of a French standardized functional development assessment, "the neuro-psychomotor functions in 
children" battery (NP-MOT) (Vaivre-Douret, 2006). According to the test manual, overall testretest reliability is good, ranging from $70 \%$ to $98 \%$. Correlation coefficients for the NP-MOT battery with the LOMDS (Lincoln-Oseretsky Motor Development Scale, Rogé, 1984) similar to the Bruininks-Oseretsky Test of Motor Proficiency (BOTMP, 1978) for upper-limb coordination, balance and bilateral coordination subtests were found to be .72 to .84 . Regarding specificity, the NP-MOT battery is a standardized normative instrument with identical subtests for any age (with expected saturation for subjects aged 8 years or more) to measure developmental maturation with qualitative (movements) and quantitative (speeds) assessments for each item of each function: neuromuscular examination, gross motor-control tasks, laterality, praxis, digital gnosis, manual dexterity, body spatial integration, rhythmic tasks, auditory-attentional task (see Appendix for tasks description). Neuro-developmental maturation depends on the neurological mechanisms on which the function is based. Its assessment also enables detection of minor neurological signs, the so-called soft-signs (Bert \& Touwen, 1979; Vaivre-Douret, 2006).

Neurovisual examination. A set of electro-physiological visual tests were performed including electro-retinogram, visually evoked potentials and motor electro-oculogram in order to study the sensory and visual motor pathways. For the electro-oculogram (EOG), skin electrodes provided by Metrovision $(9)$ (59840 Perenchies, France) were used to study vertical and horizontal eye movements, as in a study already conducted in our child patients (Ingster-Moati et al., 2005, 2009). Calibrated amplitude and velocity of horizontal and vertical smooth pursuit (SP) were compared to normative values obtained in a normal sample (Ingster-Moati, Vaivre-Douret, Delouvrier et al. 2005). A photopic full-field Electroretinogram (ERG) was performed to check the integrity of retinal function. The children's pupils were not dilated. The Metrovision@ apparatus was used for stimulation and recording. The input impedance of the amplifiers was 1000 G $\Omega$. During the ERG recordings, the child continued to sit comfortably and his or her head rested on a head restraint; the child was instructed to look straight forward and open his or her eyes. Patterns of visually evoked potentials (VEP) were also recorded. The stimuli consisted of a highcontrast black and white check of 60,30, and 15 pattern element size in minutes, in a field of $30^{\circ}$ as recommended by the International Society for Clinical Electrophysiology in Vision (ISCEV) standards. Amplitudes and peak latencies of ERG and VEP were compared to normative values.

\section{Anatomical MRI: Brain Imaging: T1, T2, and FLAIR}

Anatomical MRI was performed with a 1.5 Tesla (Signa General Electric) scanner using the following sequences: 3D T1-weighted sequence, axial and coronal FSE T2-weighted imaging and coronal FLAIR sequences.

\section{Statistical Analysis}

Association between variables were tested using chi-squared statistics. In the case of a quantitative variable (e.g., IQ), between groups comparisons were based on one-way ANOVA; post-hoc tests (HSD Tukey) to assess pair-wise differences are also reported. In all cases, statistical significance was assessed at the 5\% level. In order to validate our clinical results, we used two statistical approaches, Factor analysis, and Cluster analysis. These enable the underlying structure to be found for the variables and the subjects, respectively. It should be noted that for cluster analysis, 
we were not considering the clinical label associated with each subject. Instead, we sought to identify distinct clusters of subjects, assuming they are randomly drawn from a population. We then aimed to compare this "naïve" solution with that obtained from purely clinical expertise. All analyses were carried out with SPSS (Release 16, 2007, Chicago: SPSS Inc) for Factor analysis and the R statistical software (2.7, Development Core Team, 2008) for cluster analysis.

\section{RESULTS}

The results are studied and analyzed from three approaches: descriptive, clinical, and statistical, in order to examine the semiology and the nature of DCD in children, to characterize the etiology and to identify the profile patterns of subtypes. First, all variables are transformed into scores based on the means and standard deviations of the national norms.

\section{Descriptive Analysis}

Concerning the pregnancies related to our sample, 35\% $(n=15)$ of the children are born by caesarean delivery and for $40 \%(n=17)$ particular medical circumstances were noted including three induced labours, three cases of umbilical cord entanglement, two detachments of the placenta, two forceps deliveries, one breech delivery, one neonatal jaundice, one hypertension, one pneumothorax, one oligo-amniosis, one heart disorder. Overall, there are 67\% $(n=10)$ of the caesarean that are motivated by medical circumstances.

In the area of motor development, $77 \%(n=33)$ of the children are sitting in the normal range of developmental scale (acquired before 9 months), 14\% $(n=6)$ are borderline (on the limit) and 9\% $(n=4)$ are delayed beyond 9 months for sitting. For walking, 67\% $(n=29)$ are in the normal range, $19 \%(n=8)$ are borderline and $14 \%(n=6)$ are late walkers after 18 months. Furthermore, $65 \%(n=28)$ do not crawl, $21 \%(n=9)$ of the children present a delay in language (after 3 years), 35\% $(n=15)$ present a refraction disorder (myopia, astygmatism, hypermetropia), and 19\% $(n=8)$ present ENT disorders.

Difficulties with Lego blocks and completion of puzzles are reported by parents for $74 \%(n=32)$ and $79 \%(n=34)$, respectively. Overall, $28 \%(n=12)$ of the children have language learning disabilities and $88 \%(n=38)$ have school failure in mathematics, mainly for geometry or setting out sums correctly in arithmetic. We also noted that $88 \%(n=38)$ have difficulties with handwriting. Impaired handwriting was characterized by spatial disorientation with letters irregular in shape and in relation to the lines, and sentences were not organized on the page, with spacing problems. Their writing is poor and they had more difficulty copying.

The mean for full IQ (FIQ) is $97.4( \pm 23.9)$, for verbal IQ (VIQ) it is $103.6 \pm 22.9$, for performance IQ (PIQ) it is $88.7 \pm 22.7$. There is a significant difference between VIQ and PIQ scores $(t(42)=6.45, p<.001)$ : VIQ is generally 15 points higher $(95 \%$ confidence interval, [10.2;19.5]) than PIQ. In our sample a majority fails (score below 11/19) for subtests in the WPPSI-R, such as Geometric Design (70\%), or Block Design (70\%), Picture Completion (50\%) and Arithmetic (80\%) and in WISC-III with the subtests Object Assembly (73\%), Block Design (73\%), Picture Arrangement (50\%), Coding (63\%), and Arithmetic (50\%).

The main results for the neuropsychological assessments are summarized in Table 1 as frequency of failure estimated from the whole cohort. 
TABLE 1

Distribution of Failure in the Main Tasks for Neuropsychological and Neuro-Psychomotor Assessment

\begin{tabular}{|c|c|}
\hline Task & Proportion of failure $(n)$ \\
\hline Visual motor integration & $88 \%(38)$ \\
\hline Visual spatial structuration & $86 \%(37)$ \\
\hline Visual construction & $72 \%(31)$ \\
\hline Visual spatial attention & $72 \%(31)$ \\
\hline Executive functions & $19 \%(8)$ \\
\hline Handwriting & $88 \%(38)$ \\
\hline Dysgraphia & $19 \%(8)$ \\
\hline Visual perception & $21 \%(9)$ \\
\hline Language disorder (reading/spelling) & $28 \%(12)$ \\
\hline Articulation & $7 \%(3)$ \\
\hline Dyslexia & $9 \%(4)$ \\
\hline Expressive dysphasia & $5 \%(2)$ \\
\hline Hyperkinesia & $19 \%(8)$ \\
\hline Auditory memory & $30 \%(13)$ \\
\hline Working memory & $37 \%(16)$ \\
\hline Visual spatial memory & $51 \%(22)$ \\
\hline Kinaesthetic memory & $14 \%(6)$ \\
\hline Hypotonia (axial/limbs) & $28 \%(12)$ \\
\hline Motor pathway disorder (mild sign) & $35 \%(15)$ \\
\hline Synkinetic movements & $63 \%(27)$ \\
\hline Dysdiadochokinesis & $47 \%(20)$ \\
\hline Homogeneous tonic laterality & $23 \%(10)$ \\
\hline Global balance & $33 \%(14)$ \\
\hline Static balance & $42 \%(18)$ \\
\hline Dynamic balance & $63 \%(27)$ \\
\hline Postural control & $42 \%(18)$ \\
\hline Upper/lower limbs coordination & $49 \%(21)$ \\
\hline Standing tone & $44 \%(19)$ \\
\hline Digital praxia & $60 \%(26)$ \\
\hline Bimanual dexterity & $56 \%(24)$ \\
\hline Gesture slowness & $60 \%(26)$ \\
\hline Imitation of gestures & $56 \%(24)$ \\
\hline Representational gestures & $10 \%(4)$ \\
\hline Orofacial praxia & $33 \%(14)$ \\
\hline Digital perception & $49 \%(21)$ \\
\hline Body spatial integration & $70 \%(30)$ \\
\hline Manual dexterity & $44 \%(19)$ \\
\hline Auditivo-visual-kiaesthetic rhythmic tasks & $7 \%(3)$ \\
\hline Auditivo- perceptivo-motor rythmic adaptation & $49 \%(21)$ \\
\hline Auditive attention & $37 \%(16)$ \\
\hline
\end{tabular}

In neuropsychological evaluation, most of children fail in visual-perceptual-motor tasks and on the visual motor integration test (Beery, 1982) were two years below the expected standard ( \pm 1 year and 6 months). Regarding the visual spatial structuring test, $60 \%$ were below $2 S D$, for the visual constructive test children had about 22 months' delay \pm 18 months, and for the visual 
spatial attention test only 35\% $(n=14)$ had strategic difficulties. Few children $(19 \%, n=8)$ have difficulties with executive functions, such as mental planning, with a delay of around one year ( \pm 30 months). Handwriting is difficult in nearly all children, involving immaturity of motor control gesture or/and irregularity in spacing or shaping letters and in keeping to the line but there are only $19 \%(n=8)$ presenting with dysgraphia. There were few visual perception difficulties.

The neuro-psychomotor functions battery (see Table 1 for details) for neuromuscular tone examination show some hypotonic children and some mild signs of motor pathway (pyramidal tract) disorder, with $19 \%(n=8)$, bilateral and 15\% $(n=6)$ left-sided, none on the right side. Tonic laterality is homogeneous for only $23 \%(n=10)$ of the children but $70 \%(n=30)$ have a right-hand tonic laterality in the upper-limbs and $7 \%(n=3)$ left-hand. Laterality is for the majority right-handed in the sample. Spontaneous gesture laterality is right-handed for $67 \%$ $(n=29)$ and left-handed for $19 \%(n=8)$. Usual laterality is right-handed for $70 \%(n=30)$ of the children and left-handed for $19 \%(n=8)$ whereas psychosocial laterality is right for $63 \%$ $(n=27)$ and left for $12 \%(n=5)$. The three lateralities (spontaneous, usual, and psychosocial) are homogeneous, that is, on the same side for $63 \%(n=27)$ of the children. Body spatial integration is appropriate for age for $30 \%$, with $67 \%(n=29)$ succeeding in relation to self, $26 \%$ $(n=15)$ in relation to others, $42 \%(n=18)$ in relation to objects and map. Rhythmic tasks are generally well-performed in auditivo-visual-kinaesthetic tests but the spontaneous rate is slow for $51 \%(n=22)$ (Table 1$)$.

Neurovisual results showed difficulties for $37 \%(n=16)$ of the children in horizontal pursuit and for $70 \%(n=30)$ in vertical pursuit; $42 \%$ of the subjects $(n=18)$ show either horizontal or vertical pursuit impairment, while $26 \%(n=11)$ are affected for both items. VEP is abnormal for $14 \%(n=6)$ of the children with P100 peak latency increased. ERG is normal for all children.

Anatomical MRI is performed for 39 children because of 4 parental refusals. MRI is rated as abnormal in 15 children. The abnormalities are heterogeneous and non-specific: posterior peri-ventricular white matter anomalies with anomalies of left hyppocampus $(n=1)$; unilateral ventricular dilatation with small left hyppocampus $(n=1)$; ventricular dilatation and white matter posterior hyper-intensities with anomalies of hyppocampus $(n=1)$; hyper-intensity on T2 and Flair in the left pallidum $(n=1)$; multiple punctate white matter hyper-intensities on T2 and FLAIR sequences and dilated Virshow-Robin spaces $(n=2)$; dilated Virshow-Robin spaces $(n=3)$; small hyppocampus $(n=1)$; dysmorphism of the corpus callosum $(n=1)$ and non-specific cysts $(n=4)$ (ponto-cerebellar cyst, retro cerebellar cyst and peri-ventricular with dilated Virshow-Robin spaces, temporal arachnoidian cyst, frontal archnoidian cyst with multiple hyper-intensities in posterior white matter $(n=1)$. Among the 39 MRI scans collected during this study, $24(62 \%)$ are considered to be normal.

\section{Inferential Clinical Analysis}

Inferential clinical analysis made it possible to distinguish different dyspraxia subtypes (Figure 1). Supported by the clinical literature on apraxia and dyspraxia previously described, assessment of ideomotor dyspraxia includes non-habitual movements or non- meaningful gestures (Bergès, \& Lézine, 1965; Ayres, 1972; Cermak, 1985; Dewey \& Kaplan, 1994) and assessment of repetitive et alternating movement sequencing tests (Denckla, 1974; Dewey, \& Kaplan, 1994). Here a child scoring one standard deviation or more below the mean on both 
tests, imitation of gestures (Vaivre-Douret, 2002) and digital praxis (NP-MOT), was considered in our sample as having ideomotor dyspraxia.

Few studies have described visual spatial or visual constructional dyspraxia in children compared to adult apraxia. The main test for children used to identify the visual motor subtype, similar to visual spatial disability, is the VMI of Beery (1982; Hoare, 1994; Macnab et al., 2001). We used different visual-perceptual-motor tests to clarify the nature of the visual motor impairment. A child who was at least one standard deviation below the mean on both tests, VMI (Beery, 1982) and Rey's complex figure (1959) was considered as having visual spatial dyspraxia. If the score on the block design in Khos's Test (1972) and the block design score in the Wechsler tests (WPPSI or WISC-III) were both one standard deviation below the mean, the child was considered as having visual constructional dyspraxia because these tasks require an object of construction to be handled in order to represent the visual spatial design. Blocks tasks involve also visual spatial processing but also required blocks to be assembled in three dimensions to form a global design. In addition, we assessed dressing-skill dyspraxia if there had been at least two failures in the relevant questionnaire items.

\section{The Subtypes of Clinical DCD Identified}

Ideomotor dyspraxia (IM). The mean age of this group was 8.2 years $( \pm 2.6)$ with $12 \%(n=5)$ of the children. We found between 80 and $100 \%$ abnormalities for crawling, digital praxis, praxic slowness, imitation of gestures, digital gnosis, dynamic balance (with $60 \%$ of postural control difficulties), body spatial integration, handwriting (but not dysgraphia), hypotonia $(60 \%)$, abnormalities in standing tone and homogeneous tonic laterality (60\% for each), and visual pursuits (vertical and horizontal $60 \%$ and $40 \%$ ). No impairment of the pyramidal tract motor pathway or of manual dexterity was found, nor any visual-perceptual-motor or VEP disorder (Figure 1). The significant failure variables $(\mathrm{p}<.05)$ compared to VSC dyspraxia are digital praxis, imitation of gestures and digital gnosis.

Visual spatial and visual constructional dyspraxia (VSC). The mean age of this group was 8.9 years $( \pm 3.0)$ with $44 \%(n=19)$ of the children. We found abnormalities in $100 \%$ of the children for puzzles, for visual motor integration and for visual spatial structuring, $79 \%$ for Lego blocks, $89 \%$ for arithmetic, $84 \%$ for visual spatial constructional tasks and handwriting (26\%, $n=5$ dysgraphia), and $68 \%$ for vertical pursuit. In addition, $53 \%$ were found to present anomalies in visual refraction. In this group $5 \%(n=2)$ of the children showed only a pure form of visual spatial dyspraxia without constructional trouble. Compared to IM group, the significant failure variables $(\mathrm{p}<.05)$ are puzzles, Lego blocks, visual motor integration, visual spatial structuring, and visual spatial constructional.

Mix dyspraxia (MD). (including ideomotor and visual spatial and/or visual constructional with motor coordination and neuropsychological co-morbidities).

The mean age of this group was 7.7 years $( \pm 1.5)$ with $44 \%(n=19)$ of the children. All abnormalities were noted, with a minimum of $10 \%$ of failure up to $100 \%$. They are reported as for the preceding subtypes in Figure 1. Compared to the aforementioned results, the most significant impairments (30\% more of failure compared to IM or VSC dyspraxia) are: coordination between upper and lower limbs (84\%), bimanual dexterity (84\%), synkinesis (89\%), manual dexterity (79\%), dysdiadochokinesis (74\%), oro-facial praxia (58\%), executive function (37\%). 


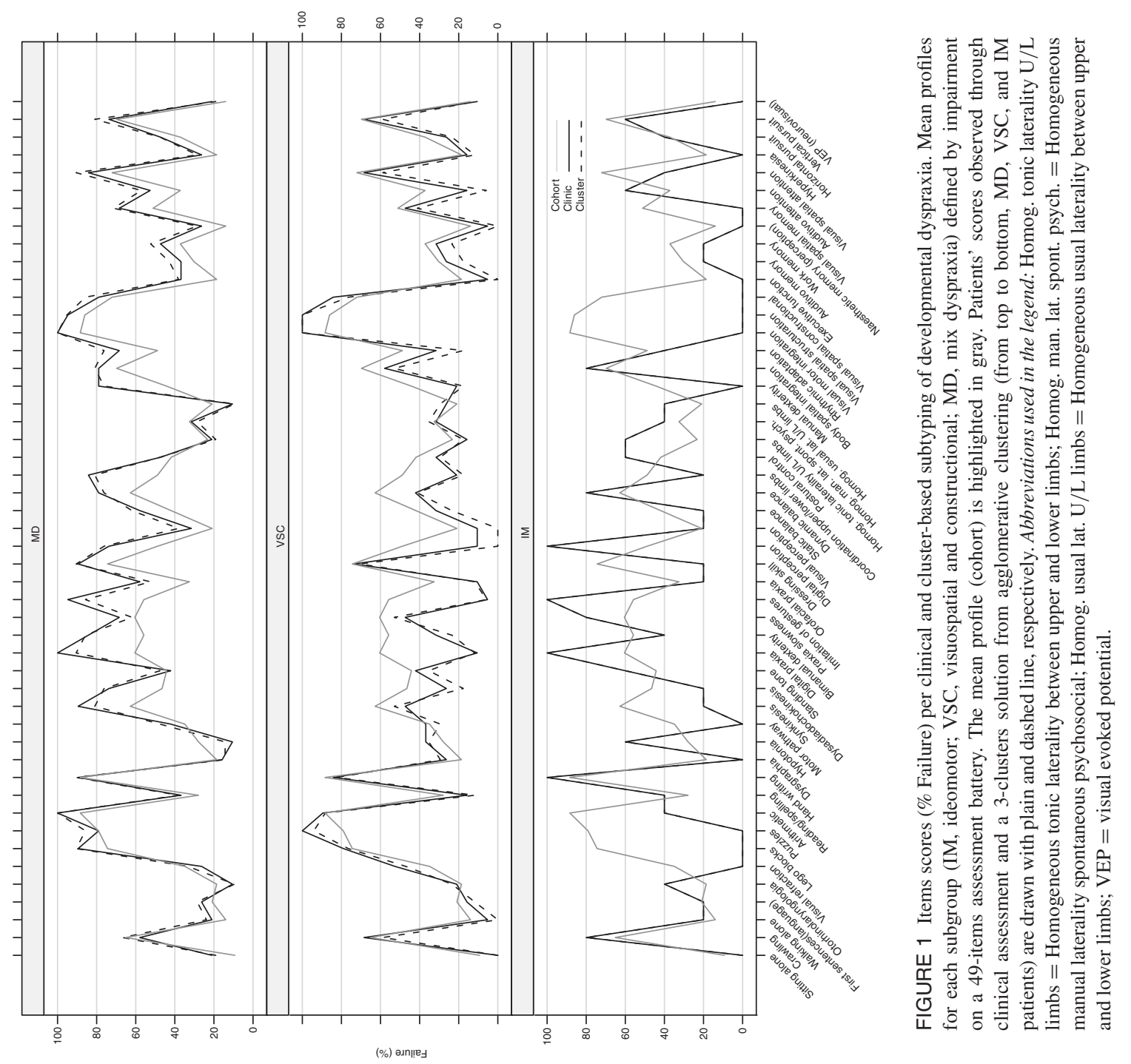


In $18 \%(n=7)$ of our sample it was noted that the motor coordination disorder (gross motor, bimanual, and manual dexterity) was not systematically associated with dyspraxia in visual spatial structuring and visual constructional dyspraxia subtype nor in visual spatial dyspraxia subtype. Moreover, there was no visual spatial structuring, visual constructional or visual motor-integration disorder in ideomotor dyspraxia.

Comparison of IQ between the three groups of dyspraxia showed significant differences, FIQ (respectively for IM $=109$ and VSC $=108$ ) was significantly lower (between-groups ANOVA, $F(2,37)=6.91, p=.003)$ for $\mathrm{MD}(\mathrm{FIQ}=84)$, as was VIQ $=90(F(2,37)=7.94, p=.0013)$ with, respectively, for IM $=112$ and $\mathrm{VSC}=115$ and $\mathrm{PIQ}=77(F(2,37)=6.38, p=.004)$, in comparison to the two other groups ( $\mathrm{IM}=107, \mathrm{VSC}=96)$. Post-hoc comparisons indicate that these differences were found to apply to each group comparisons (Tukey HSD, all $p<.05$ ). The mean difference between VIQ and PIQ was always in favor of VIQ for all dyspraxia groups, but visual spatial/visuo-constructional and ideomotor dyspraxia showed a larger mean differences with 19 points and 18.5 points, respectively, while we found only 13 points difference for mix dyspraxia pointing to greater impairment in this case. There were certain subtests in the WPPSI and WISC-III in which children frequently failed.

Some strong significant correlations between items and dyspraxia subtypes are interesting from a clinical point of view. Only the following items were not significant at the 5\% level: crawling, first sentences (language), handwriting, standing tone, homogeneous psycho-social laterality, body spatial integration, auditory memory, vertical pursuit, and VEP (neuro-visual). Although tests were not corrected for multiple comparisons, it should be noted that significant differences between subgroups were generally associated with $p$-values $<.00001$. Given the number of univariate tests, using a Bonferroni correction still yields significant $p$-values at the 5\% level.

We compared the profile of subjects with normal and abnormal MRI $(n=9)$ scans within each group, excluding IM patients since they all have a normal MRI scan. It should be noted that these comparisons are largely limited by the sample size and should be viewed as purely descriptive. For the VSC group, we noticed that the following items consistently differ according to MRI status: dynamic balance, coordination between upper and lower limbs, postural control, homogeneous tonic laterality, and usual laterality between upper and lower limbs showed a higher failure rate for normal MRI, whereas the reverse was observed for crawling and first sentences. For the MD group, subjects with abnormal MRI were more often in failure for first sentences. Overall, for subjects with abnormal MRI, regardless of the group of dyspraxia, there seem to be differences showing that there are more frequent language learning disabilities, more memory disturbances (auditory, working and kinaesthetic memory), more disorders in visual perception and executive functions, in auditory attention and in VEP.

\section{Factor Analysis}

Factor analysis has the ability to draw together highly correlated variables and helps to identify underlying, not directly observable constructs. Our aims were initially to uncover a set of meaningful latent factors, through the correlation structure of our 50 descriptors, that might facilitate further interpretation of the main characteristics of dyspraxia. All but one variable (Neuro-visual ERG) were submitted to a Factor Analysis, followed by a VARIMAX rotation so as to facilitate the interpretation of the variable loadings on the factorial axes. As there are 43 subjects in 
this study, only the first 43 principal components (out of 49) were retained. This factor model should be viewed as a semi-exploratory tool to filter out the most relevant descriptors among the entire set of variables. The results suggest that three factors might well describe the underlying structure, as evidenced by their eigenvalue greater than 1 and their associated communalities. In the description of factorial structure below, we are considering a cutoff value of .300 (in absolute value) for variable loadings. With such a cutoff, any highlighted variables are expected to explain a minimum proportion of $9 \%$ of shared variance with the underlying dimension. This value is also just above the value of a reliable correlation coefficient for that sample size. It should be noted that with such a restriction, some of the variables (e.g., Crawling, ENT disorders) still cannot be included in the constructs under consideration. These are mainly secondary variables.

As can be seen from Table 2, Factor 1, which accounts for about $20 \%$ of the total score variance, is associated with variables about dominant abnormalities in muscle tone and motricity; we consider this factor to reflect underlying tonico-sensory-motor disabilities. Factor 2 is associated with dominant abnormalities in visual-spatial and visual-constructional tasks, hence it is considered to reflect underlying visuo-spatial-motor disabilities. Finally, anomalies in learning abilities are reflected in Factor 3. For the first principal factor, digital praxia, dynamic balance, coordination between upper and lower limbs, and dysdiadochokinesis have among the highest loadings, as well as communalities (i.e. unique variance or variance adjusted for all other variables). Obviously, these variables appear to be good proxies for dyspraxia but if we examine the structure of factor 2 more closely, we can see that the most significant variables associated with it are: puzzles, arithmetic, visual motor integration, and visual spatial structuring. These are specific descriptors for visual spatial and visual constructional dyspraxia subtypes, as described in the preceding section.

\section{Cluster Analysis}

It has been demonstrated that cluster analysis (Hoare, 1994; Macnab et al., 2001) is a useful tool in the identification of subtypes of DCD. However, According to Macnab and colleagues (2001) the selection of variables should be guided by a clearly stated theoretical framework in order to guide the interpretation of the results. We retained 49 variables, as before, in order to understand the nature of the disorder. Various clustering strategies have been proposed, depending on the kind of data at hand and theoretical assumptions for the way individuals should be grouped together (e.g., Forgy, 1965; Hastie, Tibshirani, \& Friedman, 2001). We primarily used an agglomerative (hierarchical) clustering procedure based on squared Euclidean distance and Ward's criteria to partition the dataset into subgroups whose characteristics might well describe the typology observed through clinical inspection of the subjects.

Inspection of the dendrogram leads to the selection of 3 clusters comprising $n=5,21$, and 17 subjects. For each cluster, we estimate the frequency of failure for each item considered previously and compare these frequencies with those defined by the clinical diagnosis. This allows us to identify each cluster with one of the three groups of patients considered so far. Comparing the profile for the clinical and cluster classifications shows that they agree very well for each type of dyspraxia, and only four subjects $(9 \%)$ were misclassified. It is worth noting that the five subjects originally classified as suffering from ideo-motor dyspraxia belong to the same cluster. Misclassification only occurs for visuo-spatial $(n=3)$ and mix dyspraxia $(n=1)$ but this is less surprising as only ideo-motor dyspraxia clearly differs from the other two (see Figure 1). 
TABLE 2

Factors Extraction Using Principal Components and VARIMAX Rotation

\begin{tabular}{|c|c|c|c|c|}
\hline \multirow[b]{2}{*}{ Variable } & \multicolumn{4}{|c|}{ Factor Loadings } \\
\hline & $h^{2}$ & $\begin{array}{c}\text { Factor } 1 \\
(18.5 \%)\end{array}$ & $\begin{array}{c}\text { Factor } 2 \\
(1.1 \%)\end{array}$ & $\begin{array}{c}\text { Factor } 3 \\
(9.2 \%)\end{array}$ \\
\hline Sitting alone & .129 & .288 & .127 & .173 \\
\hline Crawling & .090 & .016 & -.242 & .175 \\
\hline Walking alone & .258 & .481 & .158 & .048 \\
\hline First sentences (language) & .361 & .091 & .402 & .437 \\
\hline Otorhinolaryngologia & .075 & -.194 & .185 & .054 \\
\hline Visual refraction & .359 & -.202 & .190 & .531 \\
\hline Lego blocks & .352 & .184 & .558 & -.085 \\
\hline Puzzles & .641 & -.248 & .752 & .120 \\
\hline Arithmetic & .430 & .136 & .639 & -.056 \\
\hline Reading/Spelling & .265 & .144 & -.103 & .484 \\
\hline Hand writing & .216 & .338 & .059 & -.314 \\
\hline Dysgraphia & .287 & -.023 & .204 & -.495 \\
\hline Hypotonia & .111 & -.322 & -.082 & -.022 \\
\hline Motor pathway & .396 & .476 & .379 & -.162 \\
\hline Synkinesis & .268 & .512 & .073 & -.015 \\
\hline Dysdiadochokinesis & .615 & .768 & .066 & -.143 \\
\hline Standing tone & .211 & .374 & .195 & .181 \\
\hline Digital praxia & .596 & .739 & -.214 & .059 \\
\hline Bimanual dexterity & .471 & 679 & -.064 & -.074 \\
\hline Praxia slowness & .246 & .165 & -.018 & -.468 \\
\hline Imitation of gestures & .558 & .662 & -.304 & .162 \\
\hline Orofacial praxia & .428 & .494 & -.165 & .395 \\
\hline Dressing skill & .433 & .256 & .485 & -.363 \\
\hline Digital perception & .508 & .623 & -.343 & .046 \\
\hline Visual perception & .224 & .277 & -.320 & .212 \\
\hline Static balance & .477 & .521 & .047 & .451 \\
\hline Dynamic balance & .546 & .724 & -.020 & -.145 \\
\hline Coordination upper/lower limbs & .662 & .807 & .101 & .035 \\
\hline Postural control & .247 & .496 & .010 & .028 \\
\hline Homog. Laterality dyn. Upper/lower limbs & .439 & .121 & -.180 & -.626 \\
\hline Homog. Man. Lat. spont. Psych. & .340 & .145 & .072 & -.560 \\
\hline Homog. Usual lat. U/L limbs & .431 & -.043 & -.029 & -.654 \\
\hline Manual dexterity & .676 & .762 & .276 & -.140 \\
\hline Body spatial integration & .050 & .176 & .054 & .128 \\
\hline Rhythmic adaptation & .454 & .629 & .020 & .239 \\
\hline Visual motor integration & .619 & -.085 & .777 & .092 \\
\hline Visual spatial structuration & .522 & -.058 & .718 & .059 \\
\hline Visual spatial constructional & .453 & -.014 & 671 & .051 \\
\hline Executive function & .615 & .577 & .198 & .492 \\
\hline Auditivo memory & .519 & .225 & .029 & .683 \\
\hline Work memory & .533 & .337 & -.044 & .647 \\
\hline Kinaesthetic memory (perception) & .276 & .352 & -.192 & .340 \\
\hline Visual spatial memory & .338 & .389 & .388 & .190 \\
\hline
\end{tabular}


TABLE 2

(Continued)

\begin{tabular}{|c|c|c|c|c|}
\hline \multirow[b]{2}{*}{ Variable } & \multicolumn{4}{|c|}{ Factor Loadings } \\
\hline & $h^{2}$ & $\begin{array}{c}\text { Factor } 1 \\
(18.5 \%)\end{array}$ & $\begin{array}{c}\text { Factor } 2 \\
(1.1 \%)\end{array}$ & $\begin{array}{c}\text { Factor } 3 \\
(9.2 \%)\end{array}$ \\
\hline Auditivo attention & .472 & -.501 & -.208 & -.422 \\
\hline Visual spatial attention & .252 & .299 & .393 & .090 \\
\hline Hyperkinesia & .441 & .348 & .416 & .382 \\
\hline Horizontal pursuit & .236 & .472 & .025 & -.114 \\
\hline Vertical pursuit & .219 & .260 & .389 & -.025 \\
\hline VEP (neurovisual) & .117 & .039 & .240 & .240 \\
\hline
\end{tabular}

Variance accounted for by each factor is indicated in parenthesis. Communalities $\left(\mathrm{h}^{2}\right)$ are reported in the second column. To help uncovering the underlying latent structure, variable with factor loading $>.300$ have been highlighted in boldface (See text for details).

Factor 1 reflects underlying tonico-sensory-motor disabilities.

Factor 2 is associated with dominant abnormalities in visual-spatial and visual-constructional tasks.

Factors 3 reflects anomalies in learning abilities.

Considering an additional fourth cluster allows us to discriminate between subjects suffering from mix $(n=8)$ and visuo-spatial dyspraxia $(n=2)$ with respect to various forms of motor and learning abnormalities (results not shown).

In the following, we propose a short summary of the three-cluster solution. Patients included in Cluster 1 (visuo-spatial and visuo-constructive, $n=17$ ) are $9.1 \pm 2.7$ years old, with $18 \%$ females $(n=3)$. Subjects have a mean FIQ of $111.9 \pm 19.7$, with a VIQ of $118.4 \pm 17.5$ and a PIQ of $99.7 \pm 20.7$. For Cluster 2 (mix dyspraxia, $n=21$ ), we note a mean age of $7.3 \pm 1.5$ years, with $10 \%$ females $(n=2)$. Subjects have a mean FIQ of $83.0 \pm 21.2$, with a VIQ of $89.6 \pm 20.7$ and a PIQ of $75.5 \pm 18.5$. Finally, subjects in Cluster 3 (ideo-motor dyspraxia, $n=5$ ) have a mean age of $8.2 \pm 2.6$ years, with $60 \%$ females $(n=3)$. Subjects have a mean IQ of $109.0 \pm 4.9$, with a VIQ of $111.6 \pm 9.4$ and a PIQ of $106.6 \pm 11.3$. Again, IQs differ significantly among the three groups (all $p<.001$ from separate Between-group ANOVAs). If we only consider the additional fourth cluster ( $n=10$, mean age $7.8 \pm 1.2$ years, $10 \%$ females) derived from the second analysis, subjects have an FIQ of $78.6 \pm 25.8$ (VIQ, $85.9 \pm 25.4$, PIQ, $74 \pm 20.5$ ) which confirms their similarities with the mix dyspraxia subgroup.

\section{DISCUSSION}

An important result of this study is that is has shown the validity and consistency of two approaches, clinical and statistical. The clinical approach was based on a theoretical position and on our own experience in identifying developmental dyspraxia in DCD groups, using an extensive battery of tests focused on academic, language, cognitive, visual-spatial, and visual-motor perception skills, and a complete battery of neuro-developmental psychomotor tests, including motor coordination and neuromuscular tone examination. We also performed neuro-visual examinations examinations in addition to NP-MOT assessment scale (VaivreDouret, 2006). 
It should be noted, however, that we did not use a standard instrument, such as BOTMP (Bruininks, 1978). Clearly, the indirect correlation (via LOMDS) between the NP-MOT and BOTMP instruments, with the latter being considered as a reference tool for establishing diagnostic criteria with respect to DCD, is not sufficient to provide clear evidence of the diagnostic properties of the NP-MOT. Although we never estimate the shared variance between NP-MOT and BOTMP, we consider that the NP-MOT yields a classification that is in agreement with both DSM-IV criteria (presence of DCD, yes or no) and performance-centric assessment provided by the BOTMP. Furthermore, NP-MOT allows a finer assessment of motor disorder (compared to criteria A of DSM-IV), with both a qualitative and quantitative approach. Hence, it is very unlikely that we overestimate DCD prevalence with our instrument. Moreover, our instrument allows to better delineate individual profile within the same DSM category, in our case DCD.

\section{Subtype Analysis}

The results of our study showed that children with DCD could be classified into at least three clinical subtypes of dyspraxia (ideomotor, visual spatial/constructional, mix) with one more subtype, visual spatial dyspraxia, which could be isolated, or into three similar robust subtypes (clusters) that showed different patterns of performance on neuropsychological, neuropsychomotor and neurovisual measures. There was a group of children with deficit in all motor skill areas, which we named mix dyspraxia, as demonstrated in the majority of studies on DCD. This group combines the two other "pure" subtypes of dyspraxia we have identified (IM and VSC) and entails co-morbidity with other abnormalities.

It is, however, interesting to analyse the variables of the "pure" forms of dyspraxia whatever the group (visual spatial, constructional, ideomotor without including mix dyspraxia (Figure 1, dashed and dotted lines), as there are variables that are not markedly involved $(>30 \%)$. They therefore appear to be co-morbidites of the abnormalities most often found in mix dyspraxia with a spectrum of non verbal learning disorder according to Rourke (1995). These co-morbidities are, from a neuropsychological viewpoint, (1) learning difficulties in areas such as language, writing, executive function, cognitive-auditory memory, kinaesthetic memory, auditory attention, VEP; (2) behavioral, such as hyperkinesis. In addition, there are few disorders of hemispheric organization related to laterality, which remains, as in the general population, right-dominant. When disorders do occur, they are related to distal spaticity of gastrocnemius muscle with axial hypertonia, hemiparesia, or axial hypotonia.

Regarding the milestones of motor development, sitting or walking at a late stage are fairly infrequent, but it is important to note that a large number of subjects are borderline to normality (14\% for sitting and 19\% for walking) or development is delayed (9\% for sitting and 14\% for walking). Crawling is the motor stage that is the most often not acquired (65\%). Manual dexterity and bimanual dexterity are disorders present above all in mix dyspraxia. Thus if it can be considered that mix dyspraxia is an association of "pure" forms of dyspraxia (ideomotor, visual-spatial, visual-constructional), there could also be co-morbidity of neuro-developmental abnormalities characterising motor coordination disorders in this group, since these abnormalities are significantly more numerous in this group $\left(\chi^{2}\right.$ test, $\left.d f=2\right)$ : dysdiadochokinesis $\left(\chi^{2}=10,17, p<.006\right)$, orofacial praxia $\left(\chi^{2}=10.11, p<.006\right)$, coordination upper/lower 
limbs $\left(\chi^{2}=17.05, p<.0001\right)$, manual dexterity $\left(\chi^{2}=17.39, p<.0001\right)$, bimanual dexterity $\left(\chi^{2}=11.24, p<.004\right)$. Thus these variables might be authentic specific markers of motor coordination disorders and are not necessarily present in the group of pure dyspraxia.

In addition, we were able to isolate a subtype of dyspraxia involving VSC dyspraxia, which implicates few or no gross motor skill disorders (see Figure 1). This may mean that gross motor disorders are not necessarily associated with dyspraxia. We also can note that no pure form of dressing skill dyspraxia nor of oro-facial dyspraxia was found. Thus these two types of disorder could be consequences that are linked to the different types of dyspraxia identified. They do not appear to form a particular type of dyspraxia.

The neural bases involved in symptomatology of mix dyspraxia may involve the same brain areas as in "pure" dyspraxia but with associated co-morbidity enhancing the motor difficulties in hemisphere and inter-hemisphere or automatized coordination. We therefore conclude that dyspraxia can be associated with specific motor coordination disorders, on the one hand with motor pathway disorders from the motor cortex (pyramidal origin for voluntary motricity) or gross motor/fine motor deficit of coordination, and on the other with specific neuro-psychological disorders (memory, executive function, ....).

We have shown that learning disorders coexist with dyspraxia, a condition in which more language disorders are observed in IM dyspraxia compared with VSC dyspraxia, and more disorders in mathematics (geometry) for VSC dyspraxia compared to IM dyspraxia, while it is mix dyspraxia that accumulates the largest number of learning disorders.

\section{Impact of Visual-Motor Perceptual Impairments}

Finally, in all the recent studies of DCD clusters (subtypes), a group of children is consistently found with motor performance deficits in all areas, where the main feature is a severe impairment in motor coordination. The other subtypes are very heterogeneous between those studies providing an adequate range of variables.

Like the Lyytinen and Ahonen (1988) and Miyahara (1994) studies, in our sample, significant differences in IQ scores were found among subtypes, but in addition we derived similar IQ ratings from clinical and cluster analysis, and IQ was lower in the subtype with all deficits. In addition, there is often a significant difference between VIQ and PIQ, as already noted by other authors (e.g., Maeland \& Søvik, 1993) but for ideomotor dyspraxia, these differences are not significant.

The relationship between DCD and visual motor perceptional impairment is not established because only five recent studies (Lyytinen \& Ahonen, 1988; Dewey \& Kaplan, 1994; Hoare, 1994; Macnab et al., 2001; Shoemaker et al., 2001) investigated visual-perceptivo-motor tasks. Indeed, in data analysis in many studies, there is a confusion between visual-motor and visual-perceptual skills. In fact, few perceptual deficits are found. Some research has indicated that both visual (e.g., Hulme, Smart, \& Moran, 1982) and kinaesthetic (e.g., Laszlo, Bairstow, Bartrip, \& Rolfe, 1988) difficulties are common in children with DCD, but according to other studies (e.g., Hoare \& Larkin, 1991), there is no relationship between motor coordination and kinaesthetic sensitivity; in addition, in our results, $85 \%$ of the children performed equally well in auditory-visual-kinaesthetic tasks and 79\% in visual perception. Henderson, Barnett, and Henderson (1994) did not establish any significant correlation between a visual discrimination task and any of the other motor tasks. If the results obtained in recent studies on clusters using perception measures are considered, the 
studies by Hoare (1994) and Macnab and colleagues (2001) reveal a cluster in which kinaesthetic sensitivity is particularly disturbed, and two clusters where there are visuo-motor disturbances in association with dynamic coordination disorders (running), as well as one cluster where there is only a visual-perceptive disorder. Thus these results show a possible separation of perceptual functions arising from sensorial (visual, kinaesthetic) or motor (visual motor) inputs. In the results obtained by Lyytinen and Ahonen (1988) there is a similar separation between kinaesthetic, perceptual, and visual motor integration. In the study by Dewey and Kaplan (1994) who used visual-perceptual tests with a motor component, these authors evidence visual-perceptual motor disorders in all cluster except the controls. These results, in accordance with our findings, suggest that a relationship may exist between motor development disorder and visual-perceptual motor skills. As evidenced by the Factor analysis results, most of the visual-perceptual related variables used in this study load onto the same factor, visuo-spatial-motor disabilities (Factor 2 in Table 2), which means that they are inter-related (through their shared correlation with this latent composite) in some way. Dewey and Kaplan (1994) did indeed wonder "what impact visual-perceptual impairments have on children's ability to perform gestures and motor sequences." Since our results revealed few perceptive disorders (kinaesthetic or visual-perceptive) but considerable visuo-spatial motor disorders (not correlated to the other perceptive disorders) and eye pursuit disorders, it can be thought that subjects perceive efficiently, but that it is the motor action that results from the task perceived that is affected. This could take place either on the level of sensory-motor conversion of the movement. The idea that sensory-motor control and visual information processing are strongly interrelated in the brain has been advocated by several authors during the last decades. Milner and Goodale (1995) proposed a distinction between two systems of vision, one oriented toward perceptual decision, the other dedicated to action control. In addition to mediating visual processing in the direction of two distinct functional endpoints, these systems are wired in separate pathways in the brain, both starting from occipital visual areas and ending in the temporal (ventral stream) or parietal (dorsal stream) areas. The dorsal pathway has an important relationship with the oculo-motor networks, which involve the parieto-occipital lobe, the frontal lobe, the cerebellum, the basal ganglia, and the brainstem in the performance of eye movements. Although this model makes it possible to explain some interesting neuropsychological observations (e.g., in visual dysgnosia and visuo-spatial trouble), other authors have challenged this somewhat strict anatomical and functional segregation of processes related to the processing of visual inputs for perception and action (e.g., López-Moliner, Smeets, \& Brenner, 2003). It is now widely agreed that perceptual and motor-related activities interact from early stages of visuo-motor processing, and that visual interpretation and the control of goal-directed arm movements are not to be viewed as distinct units of treatment in the brain. In the Shoemaker and colleagues (2001) study using visual-perceptual and visual motor tasks, the authors concluded that the motor component present in some visual-motor integration tasks seemed to contribute more to poor performance in the DCD group than the perceptual component. These findings are in accordance with our results and the results found in the study by Van Waelvelde, De Weerdt, De Cock, and Smits-Engelsman (2004) where not all children with DCD have problems with visual-perceptual and/or visual-motor integration tasks. In this case, the visual-perceptual impairment of some of the children with DCD is not the cause of their motor impairment. The heterogeneity of the results in the studies between DCD and control groups can be explained by deficits associated with the confounding co-morbidity of developmental disorders. 


\section{Gross Motor Deficits Versus Fine Motor Deficits}

Motor deficits that we found in our study can be partly separated into gross motor and fine motor between gross motor deficits and fine motor deficits. In addition, as in the findings reported by Hoare (1994) gross motor deficits can be separated between static balance and dynamic balance impairment. Our findings show that gross motor disorders (static and dynamic) are most commonly $(p<.003)$ coordination disorders between upper and lower limbs (asynergy) found in mix dyspraxia and more indicative of problems with general control of coordination, or inter-hemispheric connectivity, that possibly characterises specific motor coordination disorders. There are indeed more frequent bi-manual dexterity disorders, and manual dexterity is related to mix dyspraxia. Finally, motor deficits evidenced in the different types of dyspraxia groups are either soft signs linked to cerebellar control (synkinesis, abnormalities in muscle tone for instance) or else they are motor disorders involving impairment of planning and programming functions (quality of the gesture) or slowness (quantitative aspect) in the act of motor execution, pointing to dysfunction of the basal ganglia and the cerebellum. When dynamic balance is affected in ideomotor dyspraxia, it appears to relate more to a problem of postural control and axial hypotonia.

Gubbay (1979) had already reported problems in handwriting. Handwriting disorders were frequent in our sample and characterized by a maladaptive grasp gesture, by poor motor control, and by irregularity, but it is distinct from dysgraphia, which was rarely found $(20 \%)$ and where there is spatial disorientation. We agree with Wann (1987) who proposed that children with DCD demonstrate poor handwriting because the underlying mechanisms in the organization of this skill are inadequate for the sophisticated movements required (Wright \& Sugden, 1996).

Visual-motor ability appears important for handwriting to assist orientation of the various grapheme strokes on the line and relative to their position on the page. All the mechanisms implicated in handwriting disorders, so long as they are not the results of a motor disorder (hemiparesia), result from difficulties in planning and programming movement, in accordance with our model. Representational gestures were not widely impaired (9\%) in our sample, which contrasts with impairment of imitation of non-representational gestures (56\%), similar to the findings of Dewey (1991). Once more, these results point to visual motor perceptual problems rather than to visual mental perceptual problems. The findings of our studies indicated that there is greater difficulty in planning bimanual asymmetrical coordination versus bimanual symmetrical coordination, with slower movement times in ideomotor and visual spatial/constructional praxia. These deficits are significantly related to dysdiadochokinesis and problems of rhythm adaptation suggesting deficits in both motor planning and programming (control) rather than impairment of the callosal pathway that transmits information between the right and left hemispheres.

The difficulties in body spatial integration that we found were essentially designation errors (left/right) on the other person's body on the one hand, and, on the other, imitation errors (including reversibility) of unilateral postures (right hand on right eye) and especially contra-lateral (right hand on left eye) crossing the median body line, although performance is better when following instructions. Again, this is in line with the idea that motor activities depend on correct integration of visual signals linked to body axis. Likewise, children may succeed in tests on orientation in relation to objects even if they fail on imitation tests. This highlights the fact that these problems are probably linked to poor integration of the spatial reference provided by the 
body axis which hinders the identification of right and left in self and hence in others. The higher the IQ, the better a child is able to compensate for difficulties by way of an internal language and mental representation enabling him to perform correct imitations with merely slower timing. Finally, we also noted more difficulties in spatial bodily integration in case of axial hypotonia.

\section{Anatomical MRI}

Although studies examining computerized axial tomography (CAT) scans of clumsy children did not find any consistent pattern of localizable areas according to Bergstrom and Bille (1978), Knuckey and colleagues (1983), they did find a higher incidence of abnormality, as we did (about $35 \%$ ). The MRI data show that subjects with abnormal MRI, regardless of the group of dyspraxia, seem to highlight more frequent language learning disabilities, more memory disturbances, more disorders in visual perception and executive functions, in auditory attention and in VEP. Subjects with abnormal MRI have different patterns of failure in the VSC and MD group, except for first sentences which has always a higher failure rate in subjects with abnormal MRI in both groups. Despite the small sample size, this suggest that incidence of abnormalities on anatomical MRI might not be specific to dyspraxia, but rather related to co-morbidity of learning disabilities.

Thus, whatever the type of analyses of profiles of dyspraxic children (clinical, cluster, MRI), the result confirm on the one hand the precision of our clinical approach, and on the other the validity of the selection criteria for our DCD sample, such as characteristics of subjects, birth term and the non-inclusion of physical abnormalities. Few studies have incorporated these individual data, as noted by Hoare (1994). A number of studies in the literature have attempted to describe subgroups or clusters within the population of children labelled DCD. However, most of these have focused on a level of analysis that ignores differences within the perceptuo-motor and cognitive domains. Moreover, these studies neglected neuromuscular tone examination, focusing on soft neurological signs (such as hypotonia, hypertonia with spasticity of the gastrocnemius, dysdiadochokinesis, synkinetic movements). Sanger and colleagues (2006) are critical of the fact that these additional signs have not yet been adequately studied in children.

The initial selection of variables is of great importance because the choice of measures has an impact on the understanding of the nature and etiology of a condition. Most studies have used screening measures of performance on certain developmental milestones derived from mix motor tests (such as M-ABC, BOTMP, TOMI), which unfortunately do not take into account qualitative and quantitative aspects of gesture in the score.

\section{Possible Neuronal Mechanisms Underlying Subtyping of Dyspraxia}

The results of the Lundy-Ekman et al. study (1991) suggested problems in clumsy children, at least for certain subpopulations of children, similar to those of patients with either cerebellar or basal ganglia dysfunction. It is known that basal ganglia are linked to the pre-motor frontal cortex. The roles of the extra-pyramidal system are also known, and they contribute to the regulation of tone and to postural adaptation and motor control. Any disorder affecting this system shows up not in a decrease in muscular strength, but in disturbances in the initiation and the regulation of gestures (control). The cerebellum plays an important part in the coordination of various neuromuscular activities and in motor learning. It has a role in the control of tone, reinforcing it and thus enabling balance on the one hand and regulation of walking on the other; it participates in 
the performance of fine voluntary gestures (precision) and in the timing of movements according to Keele, Pokorny, Corcos, and Ivry (1985) and Volman and Geuze (1998). If the cerebellum is affected, this can explain hypotonia, balance disorders, dysdiadochokinesis, and asynergy (of automatic postural responses) and it is also involved in attentional disorders (Fiez, 1996).

Thus the etiology of dyspraxia suggested by Dewey and Kaplan (1994) according to Roy's adult model, with a mainly frontal dysfunction in the frontal and parietal-occipital areas, could have a different origin in children with "pure" developmental dyspraxia because the difficulties are closer to motor planning disorder and programming disorder than to executive disorder. As suggested by the Lundy-Ekman et al. study (1991) and regarding our clinical results, we think that the etiology of developmental dyspraxia relates to the subcortical network of the brain rather than the cerebral cortex. Indeed it should be noted here that unlike adults with apraxia who have a disorder in the execution of already learned movements (Geschwind, 1975), the child with developmental dyspraxia has an impairment in the performance of planning of new skilled or non-habitual motor tasks (Ayres, 1972). Ayres emphasized the role of the tactile system in motor planning, involving dysfunction of the dorsal column medial lemniscal system, and the role of vestibular processing in gross motor dysfunction. We consider that the thalamus could explain the specific dysfunction in common to the different types of dyspraxia groups. The thalamus is the relay for all sensory pathways (with the exception of olfactory pathways) between the periphery and the cortex: (1) It is a very localised relay for the lemniscal pathways (somaesthetic, tactile and proprioceptive). Part of the thalamus (the lateral ventral posterior nucleus) (LVP) receives influx from a particular region of the opposite body half (according to the somatopia of the different body regions). Thus the thalamus plays a part in the transmission of specific somaesthetic influxes, it discriminates and selects, that is, it has a partial function of sensory integration, which could explain ideomotor dyspraxia when it malfunctions. Indeed, the LVP nucleus in turn projects to the parietal cortex and the efferent axons reach the ascending parietal circumvolution which is the primary somaesthetic zone, and where there is a representation of the body-image enabling identification of the origin of a stimulus (tactile and articulatory). Thus if incorrect somaesthetic information is transmitted by the thalamus, this can upset body representations and thereby the planning function, on account of planning motor action involving a digital perception, and digital praxia, as in imitation of gestures. In the performance of motor sequences, Ayres (1977) identified problems in tactile perception, where the deficit in body representation is related to deficits in processing somato-sensory information. (2) The thalamus in an integrator of motor function in the two man loops of extra-pyramidal control (in connection with the lenticular nucleus and the cerebellum) and cortical control of motricity, which could explain, in case of dysfunction, difficulties in visual-perceptive-motor planning or perceptive-motor planning noted in VSC dyspraxia. These are difficulties in visual-spatial and motor integration. Further to this, the ophthalmic difficulties in our sample (with 35\% presenting refraction disorder) in line with Mon-William, Wann, and Pascal (1994), cannot on its own explain the difficulty of dyspraxic children with motor control. None of the children in our sample had a retinal dysfunction, and ERG was normal. This might again be explained by the role of the thalamus which has a part known as the corpus geniculatum that receives the optical pathways and projects on the primary visual area (occipital lobe). This could explain VEP abnormalities in VSC, and especially in mix dyspraxia showing a functional integrity disorder in the afferent visual pathways, hampering the flow of information to the primary visual cortex. Concerning the maintenance of smooth pursuit eye movements, the brain must keep track of pursuit velocity to reconstruct target velocity from 
the motion of retinal images. However, a recent study by Tanaka (2005) has shown that many neurons in the ventro-lateral thalamus exhibit directional modulation during pursuit, and he concluded that the central thalamus regulates and monitors smooth pursuit eye movements. As in the study by Ingster-Moati et al. (2005), we found a significant number of children with developmental coordination disorder such as dyspraxia, who presented saccadic horizontal smooth pursuits (SP) and frequently vertical saccadic SP (Ingster-Moati et al., 2009). It appeared that vertical SP in children with DCD was impaired more often than in the normal population, even allowing for the known slowness of maturation in vertical SP (Grönqvist et al., 2006; Ingster-Moati et al., 2009). The cerebellum and the brainstem are likely to be involved in this maturation processes and to play an important role in the control of smooth pursuit eye movements via feedback loops involving the basal ganglia and thalamus. (3) By way of its non-specific nucleus, the thalamus is involved in the control of vigilance and attention along with the cerebellum, explaining difficulties noted in visual-spatial attention.

\section{Suggested Integrative Model for Developmental Dyspraxia}

Finally, our previous speculative integrative model for developmental dyspraxia (Vaivre-Douret, 2007) improved here (Figure 2) remains valid to speculate on the neural basis of the different forms of developmental dyspraxia. Dyspraxia is a disorder of the intentional gesture aimed at a target where the planning and/or programming of the movement is disturbed in specific way in the subcortical zone and the cerebellum, leading to a disorder in sensory-motor and spatial-temporal integration. Afference of perceptual functions is good in dyspraxic children, but the planning of the movement is disturbed in the basal ganglia and the thalamus, where extra-corporeal sensory-perceptual indices required for the task (tactile, kinaesthetic, visual, auditory) are integrated. In this case, incorrect information is sent to the cortex (prefrontal, parietal, temporo-occipital) and movement planning is disturbed and it cannot be automatically corrected because there is dysfunction of the cerebellum-thalamus-basal ganglia circuit. However the role of movement planning is to provide the spatial and temporal parameters for movement with the back-up of retro-actions (controls) that are proprioceptive (information on the position of the hands in space and the displacement of the body) and vestibular (information in the inner ear), which participate in the visual and auditory balance of the body when it changes its position. This circuit projects the influx onto the pre-motor cortex (movement coding) and the motor cortex enabling the execution of movement (Figure 2). The basal ganglia thus have an important role in preparing or initiating movement and in suppressing undesired movements.

The nature of disorders in developmental dyspraxia is similar in both planning functions (IM dyspraxia) and programming functions (control) (VCS dyspraxia) and the etiology seems to be subcortical occurring in constitutional developmental disorder. In our sample a considerable proportion of subjects (40\%) experienced abnormalities in the neonatal period.

The executing mechanisms and the cortex seem to be intact in the "pure" dyspraxia group. In case of executive motor dysfunction, this concerns a co-morbidity of abnormalities, which can affect the motor pathway (pyramidal) in moderate manner (spasticity, hemiparesia, dysdiadochokinesis) or inter-hemisphere and automatic control, causing global coordination disorders found in mix dyspraxia. 


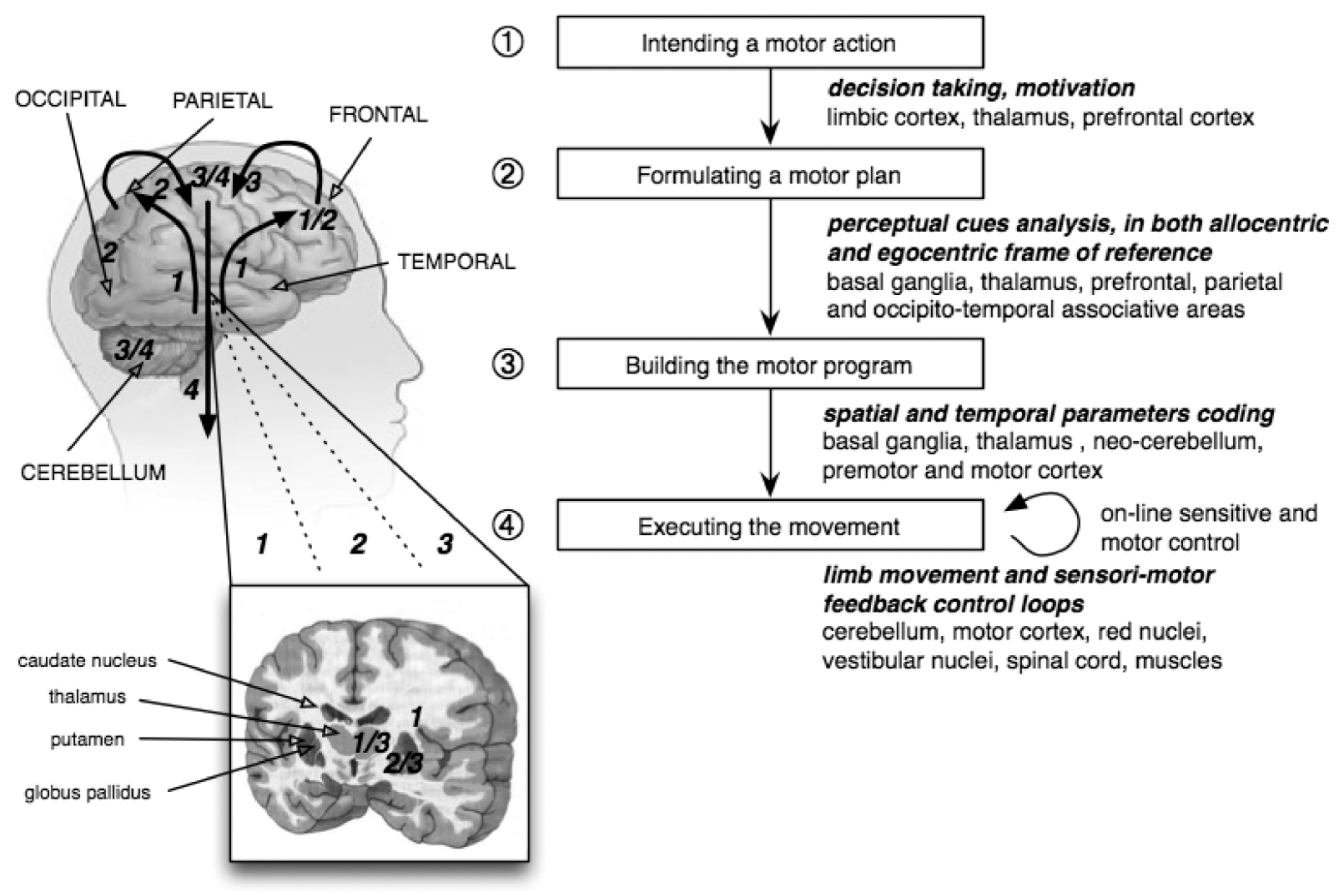

FIGURE 2 Anatomo-functional model. (Left) Anatomo-functional pathways sustending perceptual and motor functions, as well as perceptivomotor coordination. (Right) Hypothetical workflow for the planning and execution of volitional movements.

\section{CONCLUSION}

The results of the present study constitute a better understanding of the nature and etiology of the different forms of dyspraxia in DCD group, and they have enabled the definition of subtypes of "pure" dyspraxia within a population of DCD children. Correlation analysis is the prime tool for investigating associations among different deficits occurring in DCD. The patterns of abnormalities in the different categories of dyspraxia evidenced by this study, also evidenced in cluster analysis, constitute an important contribution for clinicians and researchers to obtain specific diagnostic criteria. We were able to isolate specific motor disorders in the subgroup of mix dyspraxia that are characteristic of specific motor coordination disorders. We refer to the activation of selected muscles in response to demands of voluntary movements (in manual dexterity, coordination of upper/low limbs, orofacial praxia, diadochokinesis). Thus, "pure" developmental dyspraxia may be distinct from specific motor coordination disorders. Moreover, in the case of motor coordination disorder, it can be associated with more numerous neuropsychological disorders and soft neurological signs.

On the basis of the findings in this study, we can conclude that planning and programming are the core problems of children with developmental dyspraxia, and that this should not be confused with problems of motor execution, or of inter-hemisphere motor control, or with any 
visual or visual perceptual disorder. In the general assessment of children with DCD, we recommend the integration of investigations involving standardized neuromuscular tone examination, visual motor perceptual versus visual perceptual tasks, neurovisual tasks, and use of standardized development measures with qualitative and quantitative measures of coordination, praxia, laterality, gnosia (perception), and body integration. Complementary neuro-psychological tests are often required to explore associated learning difficulties (executive, memory, attentional). Indeed, the choice of appropriate measures has an impact on understanding of the nature and etiology of disorders.

Our findings constitute a major contribution to clearly define the nature of DCD, completing the DSM-IV-R definition with clinical signs criteria. Thus they should enable therapists and teachers to better understand how to help the dyspraxic child with or without motor coordination disorder. We think it is worthwhile encouraging a therapeutic approach based on cognitive strategies and/or mental imagery depending on the particular type of task, in order to lessen difficulties in motor planning and programming. This does indeed appear as one of the most beneficial therapeutic approaches from the results of the meta-analyses by Polatjko and Cantin (2005).

\section{REFERENCES}

Ajuriaguerra (de), J., Auzias, M., \& Denne, A. (1989). L'écriture de l'enfant: l'évolution de l'écriture et ses difficultés (4rd). [Child writting: follow up and difficulties]. Neuchâtel (Suisse): Delachaux \& Niestlé.

Ajuriaguerra (de), J., \& Stambak, M. (1969). Developmental dyspraxia and psychomotor disorders. In P. J. Vinken \& G. W. Bruyn (Eds.), Handbook of clinical neurology (pp. 443-464). Amsterdam, The Netherlands: North Holland.

Albaret, J. M., Carayre, S., Soppelsa, R., \& Michelon, Y. (1995). Hétérogénéité des dyspraxies de développement: tentative de classification [ developmental dyspraxia: attempt of classification]. Approche Neuropsychologique des Apprentissages de l'Enfant, 32, 61-67.

American Psychiatric Association. (2000). Diagnostic and statistical manual of mental disorders (4th ed., text rev.). Washington, DC: Author.

Amiel-Tison, C. I., Njiokiktjen, C., Vaivre-Douret, L., Verschoor, A., Chavanne, E., \& Garel, M. (1996). Relation of early neuromotor and cranial signs with neuropsychological outcome at 4 years. Brain and Development, 18, $280-286$.

André-Thomas, \& Ajuriaguerra (de), J. (1949). Etude sémiologique du tonus musculaire [Semiological study of muscular tone]. Paris, France: Flammarion.

Ayres, A. J. (1972). Sensory integration and learning disorders. Los Angeles, CA: Western Psychological Services.

Ayres, A. J. (1977). Cluster analyses of measures of sensory integration. American Journal of Occupational Therapy, 31 , $362-367$.

Beery, K. E. (1982). Revised administration scoring and teaching manual for the development Test of Visual-Motor Integration (VMI). Toronto, Canada: Modern Curriculum Pass.

Bergès, J. (1963). Neurologie du développement chez l'enfant de 2 à 7 ans. Technique d'examen. [Neurology of child development from 2 to 7 years old]. Pédiatrie, 18(3), 301-318.

Bergès, J., \& Lézine, I. (1965). The imitation of gestures. (Trans. A. H. Parmelee). London, England: The Spastics Society Medical Education and Information Unit in Association with William Heinemann Medical Books Limited.

Bergstrom, K., \& Bille, B. (1978). Computed tomography of the brain in children with minimal brain damage: A preliminary study of 46 children. Neuropediatrics, 9, 378-384.

Bert, C., \& Touwen, L. (1979). Examination of the child with minor neurological dysfunction. Spastics International Medical Publications (2nd ed.). London, England: William Heinemann Medical Books, Philadelphia, PA: J. B. Lippincott Co.

Bruininks, H. (1978). Bruininks-Oserestky test of motor proficiency. Circles Pines, MN: American Guidance Service.

Cermak, S. (1985). "Developmental dyspraxia.” In E. A. Roy (Ed.), Neuropsychological studies of apraxia and related disorders (pp. 225-248). Amsterdam, Holland: Elsevier Sciences Publishers.

Chevrie-Muller, C., \& Plaza, M. (2001). Nouvelles épreuves pour l'examen du langage (N-EEL) [News tests for language examination]. Paris, France: Editions du Centre de Psychologie Appliquée. 
De Lange, F. P., Hagoort, P., \& Toni, I. (2005). Neural topography and content of movement representations. Journal of Cognitive Neuroscience, 17, 97-112.

Denckla, M. B. (1974). Development of motor coordination in normal children. Developmental Medicine and Child Neurology, 16, 729-741.

Denckla, M. (1984). Developmental dyspraxia: The clumsy child. In M. D. Levine \& P. Satz (Eds.), Middle childhood: Development and dysfunction (pp. 245-260). Baltimore, MD: University Park Press.

Dewey, D. (1991). Praxis and sequencing skills in children with sensorimotor dysfunction. Developmental Neuropsychology, 7(2), 197-206.

Dewey, D. (1993). Error analysis of limb and orofacial praxis in children with developmental motor deficits. Brain and Cognition, 23, 203-221.

Dewey, D., \& Kaplan, B. J. (1994). Subtyping of developmental motor deficits. Developmental Neuropsychology, 10(3), 265-284.

Fiez, J. A. (1996). Cerebellar contributions to cognition. Neuron, 16, 13-15.

Ford, F. R. (1966). Diseases of the nervous system in infancy, childhood and adolescence (5th ed.). Springfield, IL: Charles C. Thomas.

Forgy, E. W. (1965). Cluster analysis of multivariate data: Efficiency vs interpretability of classifications. Biometrics, 21, 768-769.

Frostig, M. (1973). Test de development de la perception [A development test of visual perception]. Paris, France: Editions du Centre de Psychologie Appliquée.

Gauthier, L., Dehaut, F., \& Joanette, Y. (1989). The bells test: A quantitative and a qualitative test for visual neglect. International Journal of Clinical Neuropsychology, 11, 49-54.

Geschwind, N. (1975). "The apraxias: Neural mechanisms of disorder of learned movements." American Scientist, 63, 188-195.

Grönqvist, H., Gredeback, G., \& Hofsten, C. (2006). Developmental asymmetries between horizontal and vertical tracking. Vision Research, 46, 1754-1761.

Gubbay, S. S. (1979). The clumsy child. In F. C. Rose (Ed.), Pediatric neurology (pp. 145-160). London, England: Blackwell.

Hastie, T., Tibshirani, R., \& Friedman, J. (2001). The elements of statistical learning. New York, NY: Springer.

Henderson, S. E., Barnett, A., \& Henderson, L. (1994). Visuospatial difficulties and clumsiness: On the interpretation of conjoined deficits. Journal of Child Psychology and Psychiatry, 35, 961-969.

Henderson, S. E., \& Sugden, D. A. (1992). Movement Assessment Battery for Children. London, England: The Psychological Corporation.

Hoare, D. (1994). Subtypes of developmental coordination disorder. Adapted Physical Activity Quarterly, 11, 158-169.

Hoare, D., \& Larkin, D. (1991). Kinaesthetic abilities of clumsy children. Developmental Medicine and Child Neurology, $33,671-678$.

Hulme, C., Smart, A., \& Moran, G. (1982). Visual perceptual deficits in clumsy children. Neuropsychologia, 20, 475-481.

Ingster-Moati, I., Vaivre-Douret, L., Bui Quoc, E., Albuisson, E., Dufier, J. L., \& Golse, B. (2009). Vertical and horizontal smooth pursuit eye movements in children: A neuro-developmental study. European Journal of Paediatric Neurology, $13,362-366$.

Ingster-Moati, I., Vaivre-Douret, L., Delouvrier, E., Crochet, K., Orssaud, C., Golse, B., \& Dufier, J. L. (2005). Exploration fonctionnelle des voies visuelles sensorielles et oculo-motrices des enfants atteints de dyspraxie développementale [Fonctional exploration of neurovisual fonctions in dyspraxia child]. Journal Français d'Ophtalmologie, 28, 107-108.

Kaplan, B., Wilson, B., Dewey, D., \& Crawford, S. G. (1998). DCD may not be a discrete disorder. Human Movement Science, 17, 471-490.

Keele, S. W., Pokorny, R. A., Corcos, D. M., \& Ivry, R. (1985). Do perception and motor production share common timing mechanisms: A correlational analysis. Acta Psychologica, 60, 173-191.

Khos, C. (1972). Test des Cubes de Khos [Khos's block design test]. Paris, France: Edition du Centre de Psychologie Appliquée.

Knuckey, N. W., Apsimon, T. T., \& Gubbay, S. S. (1983). Computerized axial tomography in clumsy children with developmental apraxia and agnosia. Brain and Development, 5, 14-19.

Korkman, M., Kirk, U., \& Kemp, S. (2003). Developmental neuropsychological assessment manual. Paris, France: Editions du Centre de Psychologie Appliquée. 
Laszlo, J. I., Bairstow, P. J., Bartrip, J., \& Rolfe, V. T. (1988). Clumsiness or perceptuo-motor dysfunction? In A. M. Colley \& J. Beech (Eds.), Cognition and action in skilled behaviour (pp. 293-309). Amsterdam, The Netherlands: North Holland.

Lesny, I. A. (1980). Developmental dyspraxia-dysgnosia as a cause of congenital children's clumsiness. Brain and Development, 2, 69-71.

López-Moliner, J., Smeets, J. B. J., \& Brenner, E. (2003). Comparing the sensitivity of manual pursuit and of perceptual judgements to pictorial depth effects. Psychological Science, 14, 232-236.

Lundy-Ekman, L., Ivry, R. B., Keele, S., \& Woollacott, M. (1991). Timing and force control deficits in clumsy children. Journal of Cognitive Neuroscience, 3, 367-376.

Lyytinen, H., \& Ahonen, T. (1988). Developmental motor problems in children: A 6-year longitudinal study. Journal of Clinical Experimental Neuropsychology, 10, 57.

Macnab, J. J., Miller, L. T., \& Polatajko, H. J. (2001). The search for subtypes of DCD: Is cluster analysis the answer? Human Movement Science, 20, 49-72.

Maeland, A. F., \& Søvik, N. (1993). Children with motor coordination problems and learning disabilities in reading, spelling, writing and arithmetic. European Journal of Special Needs Education, 8(2), 81-98.

Mazeau, M. (1995). Déficits visuo-spatiaux et dyspraxies de l'enfant. Du trouble à la rééducation [Visua-spatial problems and child dyspraxia]. Paris, France: Masson.

Milner, A. D., \& Goodale, M. A. (1995). The visual brain in action. Oxford, England: Oxford University Press.

Missiuna, C., \& Polatajko, H. (1995). Developmental dyspraxia by any other name: Are they all just clumsy children? American Journal of Occupational Therapy, 49(7), 620-627.

Miyahara, M. (1994). Subtypes of students with learning disabilities based upon gross motor functions. Adapted Physical Activity Quarterly, 11, 368-382.

Mon-Williams, M. A., Mackie, R. T., McCulloch, D. L., \& Pascal, E. (1996). Visual evoked potentials in children with developmental coordination disorder. Ophthalmic, 16, 178-183.

Mon-Williams, M. A., Wann, J. P., \& Pascal, E. (1994). Ophthalmic factors in developmental coordination disorder. Adapted Physical Activity Quarterly, 11, 170-178.

Odédys. (2005). Outils de dépistage des Dyslexies, version 2. Grenoble, France: Cogni-Sciences IUFM.

Polatajko, H. J., \& Cantin, N. (2005). Developmental coordination disorder (dyspraxia): An overview of the state of the art. Seminars in Pediatric Neurology, 12(4), 250-258.

Porteus, S. D. (1952). Test des Labyrinthes de Porteus [Porteus test]. Paris, France: Editions du Centre de Psychologie Appliquée.

Rasmussen, P., \& Gillbert, C. (2000). Natural outcome of ADHD with Developmental Coordination Disorder at age 22 years: A controlled, longitudinal community-based study. Journal of the American Academy of Child \& Adolescent Psychiatry, 39, 1424-1431.

Rey, A. (1941). L'examen psychologique dans le cas d'encéphalopathie traumatique [Psychological examination in encephalopathy]. Archives de Psychologie, 112(28), 286-340.

Rey, A. (1959). Manuel test de copie d'une figure complexe [Manual of complex Rey's figure]. Paris, France: Editions du Centre de Psychologie Appliquée.

Rogé, B. (1984). Manuel de l'Echelle de Développement Moteur de Lincoln-Ozeretsky [Manual of motor developmental scale]. Paris, France: Editions du Centre de Psychologie Appliquée.

Rourke, B. P. (1985). Neuropsychology of learning disabilities: Essentials of sub-type analysis. New York, NY: Guilford Press.

Rourke, B. P. (1995). Syndrome of non verbal learning disabilities: Neuro-developmental manifestations. New York, NY and London, England: Guilford Press.

Roy, E. A. (1978). Apraxia: A new look at an old syndrome. Journal of Human Movements Studies, 4, 191-210.

Sanger, T. D., Chen, D., Delgado, M. R., Gaebler-Spira, D., Hallett, M., \& Mink, J. W. (2006). Definition and classification of negative motor signs in childhood. Pediatrics, 118(5), 2159-2167.

Shoemaker, M. M., Wees, M. van der., Flapper, B., Verheij-Janssen, N., Scholten-Jaegers, S., \& Geuze, R. H. (2001). Perceptual skills of children with developmental coordination disorder. Human Movement Science, 20, $111-133$.

Sigmundsson, H., Hansen, P. C., \& Talcott, J. B. (2003). Do "clumsy" children have visual deficits. Behavioural Brain Research, 139, 123-129. 
Stott, D. H., Moyes, F. A., \& Henderson, S. E. (1984). Test of motor impairment. Guelph, Canada: Brook Educational Publishing Ltd.

Tanaka, M. (2005). Involvement of the central thalamus in the control of smooth pursuit eye movements. Journal of Neuroscience, 25(25), 5866-5876.

Vaivre-Douret, L. (2002). A more robust predictor of ideomotor dyspraxia study or an alternative scoring method of the Bergès-Lézine's imitation of gestures test. Archives of Clinical Neuropsychology, 17, 37-48.

Vaivre-Douret, L. (2006). Batterie d'évaluation des fonctions neuro-psychomotrices (NP-MOT) de l'enfant [Tests battery of neuro-psychomotor functions in children (NP-MOT)]. Paris, France: Editions du Centre de Psychologie Appliquée.

Vaivre-Douret, L. (2007). Troubles d'apprentissage non verbal: les dyspraxies développementales [Non-verbal learning disabilities: developmental dyspraxia]. Archives de Pédiatrie, 14, 1341-1349.

Van Waelvelde, H., De Weerdt, W., De Cock, P., \& Smits-Engelsman, B. C. M. (2004). Association between visual perceptual deficits and motor deficits in children with developmental coordination disorder. Developmental Medicine and Child Neurology, 46, 661-666.

Visser, J. (2003). Developmental coordination disorder: A review of research on subtypes and comorbidities. Human Movement Science, 22(4-5), 479-493.

Volman, M. J. M., \& Geuze, R. H. (1998). Relative phase stability of bimanual and visuomanual rhythmic coordination patterns in children with a developmental coordination disorder. Human Movement Science, 17, 541-572.

Wann, J. P. (1987). Trends in the refinement and optimization of fine-motor trajectories: Observations from an analysis of the handwriting of primary school children. Journal of Motor Behaviour, 19, 13-37.

Wright, H. C., \& Sugden, D. A. (1996). The nature of developmental coordination disorder: Inter- and intragroup differences. Adapted Physical Activity Quarterly, 13, 357-371.

\section{APPENDIX}

Neuro-psychomotor functions in children "battery (NP-MOT)" (Vaivre-Douret, 2006)

\section{Neuromuscular Tone Examination}

This battery of neuro-developmental tests (Vaivre-Douret, 2006) includes a complete assessment of neuro-motricity based on neuromuscular examination of muscle tone, following the technique developed by André-Thomas and Ajuriaguerra (1949), Bergès (1963), and Bert and Touwen (1979). Resistance to passive movements is tested by segmented responses that are coded and compared, for instance, extensibility of shoulders, hands, trunk, adductor angles, heel-ear angle, popliteal angle, dorsiflexion angle of foot. This last measure associated with hyperextension of the trunk enables detection of a phasic stretch reflex in one or both gastrocnemius muscles, which is a mild sign of spasticity of pyramidal tract motor pathway disorder (Amiel-Tison et al., 1996). Elicitation of the knee jerk reflex is also tested. Resistance of slack or dangling hands and feet is observed in terms of amplitude. Passive tone enables detection of hypotonia or hypertonia of axis and/or limbs. Examination of the extensibility and dangling of hands and feet allow to study the resistance corresponding to the dominant side, that is tonic laterality. Tonic laterality is considered homogeneous if the resistance between upper-limb and lower-limb is normally opposed. Indeed, for a right-hander child, his right hand should show more tonicity than the left one whereas for the lower limbs the reverse applies: his left foot ensures most of the standing position.

Evaluation of active tone consists in the observation of dysdiadochokinesis and synkinetic movements (co-movements and mirror movements) such as rapid pronation and supination of the hand and forearm, repeated opening and closing of both hands, repeated opening and closing the 
mouth. Standing tone is evaluated by the child, seated on the floor, having to resist the different pushes by the examiner (right, left, backwards, forwards).

\section{Gross Motor-Control Tasks}

Gross motor control (dynamic and static) is scored for posture of the body and limbs and performance of balance. A score of general limb coordination (between upper and lower limbs) is recorded for flying jump (i.e., jump with feet together), spontaneous walk and a score for postural control on landing after a jump (landing with two feet together).

\section{Laterality}

Spontaneous gesture laterality is observed with four verbal command items where the child is asked, for example, to put one hand above the other hand, or to cross his arms, the dominant hand being always above the other. Usual laterality of upper-limb (hand), lower-limb (foot), and eyes are examined using objects. It reflects the spontaneous laterality observed in children when presented with a new object that they have to interact with (e.g., grasp an object or shoot a ball). Normally, usual laterality of upper-limb is the same as for lower-limb and is in this case considered homogeneous. Psychosocial laterality is observed from six mimes (representational gestures) performed on verbal command, similar to the test used for transitive gestures by Dewey (1993).

\section{Praxis}

Manual praxis examination consists in assessing timing and ability to sequence the performance of repetitive and alternating movements, copying the action demonstrated by the examiner using bilateral hand pronation-supination, with symmetrical and asymmetrical movements (bimanual dexterity), followed by digital praxis, for instance speed of repetitive index-finger-thumb touching for each hand; successive touching thumb to fingertips for each hand, eyes open. These tasks are similar to those used by Denckla (1974) and Dewey and Kaplan (1994).

\section{Digital Perception (Gnosis)}

Localization of digital tactile stimuli are evaluated from successful performance when the child, one hand concealed, is asked to show on the other (not to name) the concealed finger touched.

\section{Manual Dexterity}

Manual dexterity is assessed for each hand recording time and fine motor quality. It consists in putting one by one the row of twelve counters into a box placed above the row counters in the middle. Task has to be completed as fast as possible. 


\section{Body Spatial Integration}

Body spatial integration (right and left) is assessed in relation to self when pointing ("show you right arm") and verbal command with axial crossing gesture ("put your right hand on your left ear"), and to others (pointing to a doll and to the examiner and imitation with axial crossing gesture), to three objects and to a map or plan.

\section{Rhythmic Tasks}

Rhythmic tasks are performed first using the spontaneous rate of regular hand taps on the table with time recorded on 21 taps. Then, an auditory-visual-kinaesthetic adaptation task is required via imitation of patterns of hand tapping by the examiner on his own body or foot tapping on the floor. The next task consists in an auditory-perceptual-motor rhythmic adaptation. The child claps on his own his hands, attempting to match the target rate set by a metronome (at three speeds: set at 90,60, and 120). Then, the child is asked to suit his walk to the same three speeds.

\section{Auditory-Attentional Task}

Auditory-attentional tasks consist in a series of sixteen taps in a Go/No-Go task. The child taps with a stick only one time when the examiner taps twice with a stick and twice when the examiner taps once. 